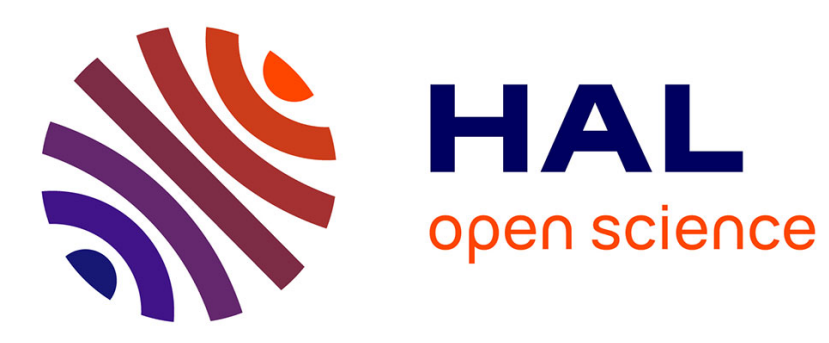

\title{
Bioadhesive Properties of Poly(alkylcyanoacrylate) Nanoparticles Coated with Polysaccharide
}

Isabelle Bertholon, Gilles Ponchel, Denis Labarre, Patrick Couvreur, Christine Vauthier

\section{- To cite this version:}

Isabelle Bertholon, Gilles Ponchel, Denis Labarre, Patrick Couvreur, Christine Vauthier. Bioadhesive Properties of Poly(alkylcyanoacrylate) Nanoparticles Coated with Polysaccharide. Journal of Nanoscience and Nanotechnology, 2006, 6 (9-10), pp.3102 - 3109. 10.1166/jnn.2006.418 . hal03196143

\section{HAL Id: hal-03196143 \\ https://hal.science/hal-03196143}

Submitted on 12 Apr 2021

HAL is a multi-disciplinary open access archive for the deposit and dissemination of scientific research documents, whether they are published or not. The documents may come from teaching and research institutions in France or abroad, or from public or private research centers.
L'archive ouverte pluridisciplinaire HAL, est destinée au dépôt et à la diffusion de documents scientifiques de niveau recherche, publiés ou non, émanant des établissements d'enseignement et de recherche français ou étrangers, des laboratoires publics ou privés. 


\section{Bioadhesive properties of poly(alkylcyanoacrylate) nanoparticles coated with polysaccharide.}

Isabelle BERTHOLON, Gilles PONCHEL, Denis LABARRE, Patrick COUVREUR, Christine VAUTHIER*

Laboratoire de Physico-chimie, Biopharmacie, Pharmacotechnie, UMR CNRS 8612,

Faculté de Pharmacie, 5 rue Jean-Baptiste Clément, 92296 Châtenay-Malabry Cedex, France

Received: 09.12.2005, Revised: 12.01.2006, Accepted: 12.01.2006

Published in: J Nanosci Nanotechnol. 2006;6(9-10):3102-9. DOI: 10.1166/jnn.2006.418.

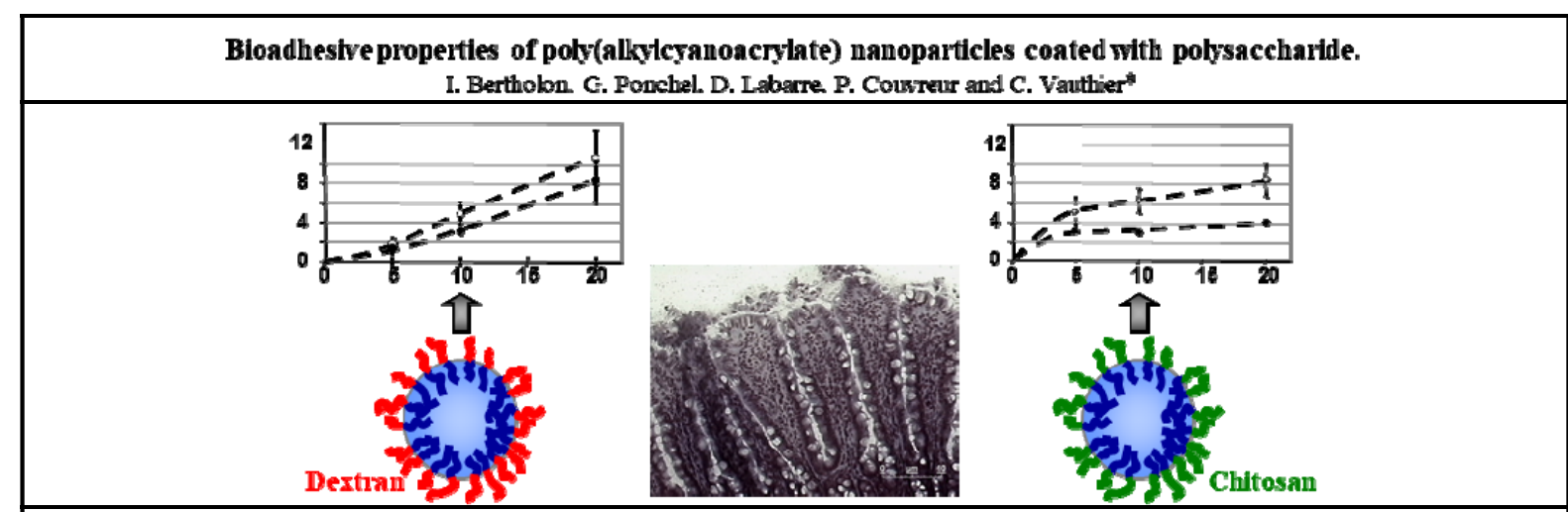

This article presents the study of the influence of nature and conformation of polysaccharide chains on the bioadhesive properties of nanoparticles coated with polysaccharides

* Corresponding Author:

Christine Vauthier

Laboratoire de Physico-chimie, Biopharmacie, Pharmacotechnie UMR CNRS 8612,

Faculté de Pharmacie, 5 rue Jean-Baptiste Clément 92296 Châtenay-Malabry Cedex, France

E-mail: christine.vauthier@u-psud.fr, Fax: 33.1.46.61.93.34. Tel: 33.1.46.83.56 03 
Bertholon et al.. J Nanosci Nanotechnol. 2006;6(9-10):3102-9. doi: 10.1166/jnn.2006.418.

\begin{abstract}
Development of bioadhesive nanoparticles is of great interest to improve drug absorption through the intestinal barrier. Various Polysaccharide-coated poly(alkylcyanoacrylate) nanoparticles were prepared. The bioadhesive properties of the nanoparticles coated with dextran or chitosan in end-on or side-on conformation were evaluated with an ex-vivo adsorption experiment on rat intestine.

Results show that diffusion of nanoparticles in mucus layer was governed by the nanoparticle diameter and isotherms of adsorption were influenced by the nature of polysaccharide used. High amount of nanoparticles coated with chitosan can be entrapped in the mucus layer even at low nanoparticle concentration in suspension. When nanoparticle concentration increased, a pseudo-plateau was reached. In the case of dextran-coated nanoparticles, linear increase of adsorption was observed and no saturation phenomenon was highlighted over the range of nanoparticle concentration used in this study. These results suggested that interactions involved in bioadhesion mechanism depended on the nature of polysaccharide. Electrostatic interactions are enhanced between chitosan-coated nanoparticles and glycoproteins of mucus leading to a saturated adsorption phenomenon whereas dextran-coated nanoparticles interacted by non-electrostatic interactions with mucus resulting in a non-saturated phenomenon. Polysaccharides grafted at the nanoparticle surface in the brush conformation appeared more favorable to promote interactions of nanoparticles with glycoproteins of mucus in comparison with the more compact loop conformation of polysaccharide chains.
\end{abstract}

Key-words: bioadhesion, gastrointestinal tract, mucus, chain conformation, nanoparticles, polysaccharides 
Bertholon et al.. J Nanosci Nanotechnol. 2006;6(9-10):3102-9. doi: 10.1166/jnn.2006.418.

\section{Introduction:}

The oral route is often preferable for drug administration because of improved compliance of the patients to their treatment, better comfort and lowered medical costs. Unfortunately, for various reasons, many compounds administered by this route are poorly bioavailable, due to low permeability of the intestinal membrane or chemical instability in the gastrointestinal tract. To solve these problems, encapsulation of fragile or poorly bioavailable molecules can improve these characteristics and thus the development of suitable encapsulation techniques is of considerable interest. Drug encapsulation in nanoparticulate systems may result in the following advantages: (i) protection of the molecules against the harsh conditins prevaling in the gastrointestinal medium, (ii) direct translocation of the particles through the intestinal barrier and (iii) immobilization of the particles at the intestinal surface due to a bioadhesion mechanism, which is of interest for prolonging the time period available for drug absorption ${ }^{1}$, ${ }^{2}$. This last property can be obtained with systems which allow an increased time of contact between delivery systems and epithelial cells, and the control of the delivery profile of the drug from the particles. Bioadhesive systems are often formulated with hydrophilic polymers which strongly interact with the mucus standing over the intestinal epithelium ${ }^{3-5}$. The mucus is mainly constituted by various glycoproteins ("mucins") composed of a protein backbone and carbohydrate side chains ${ }^{6,7}$, rendering these molecules extremely hydrophilic in nature and capable of forming coherent and elastic hydrogels. Its physiologically function is to protect the cells of the intestinal epithelium from the luminal content and catch nutriments to be absorbed. These glycoproteins are negatively charged at physiological $\mathrm{pH}$. The thickness of the mucus layer depends on the region of the intestine and decreases from 50 to $500 \mu \mathrm{m}$ in the stomach to $16-50 \mu \mathrm{m}$ in the colon ${ }^{6}$. The mucus layer is one of the barriers to drug absorption. Thus it may be interested to retain the drug as close as possible to the epithelial cells by artificial means ${ }^{8}$. To this aim, nanoparticles are able to diffuse into the mucus layer. They can be better retained at the surface of enterocytes brush border thanks to their small size compared to microparticles ${ }^{6}$. However, their retention may be further improved by coating the nanoparticles with hydrophilic polymers like chitosan or poly(ethylen glycol) to develop bioadhesive formulation.

Chitosan has been widely used in bioadhesive formulations intended to the oral route. It is known as a low toxic excipient with strong bioadhesiveness, due to the presence of positive charges which can interact with the negatively charged glycoproteins of mucus. Chitosan was 
suspected to promote the oral absorption of hydrophilic drugs by opening the tight junctions of the epithelial membrane ${ }^{1,9-11}$.

Mechanisms and parameters affecting the bioadhesive behaviour of nanoparticles in the gastrointestinal tract were investigated in several works ${ }^{12-15}$. It was proposed that bioadhesion of particles in the nano-size range resulted from two phenomenon: A diffusion of the nanoparticles occured at first from the suspension to the intestinal surface. Then the nanoparticles can interact with the surface of the intestine resulting in the immobilization of the particles on the mucosa ${ }^{5}$. Obviously, the attachment of the nanoparticles on the mucosa depends on the physico-chemical properties of the particles. For example, it has been suggested that the particles could diffuse into the mucus layer, depending on their size according to a size exclusion process ${ }^{16}$. Once in close contact with the mucus glycoproteins, various types of interactions may occur between the surface of the particles and the glycoproteins, including electrostatic or hydrophobic bonding. Although it is obvious that the bioadhesiveness of the nanoparticles on mucosa depends greatly on their surface characteristics, there is no work so far which has considered the effect of the conformation of hydrophilic polymer chains at the nanoparticles surface. Thus the purpose of our work was to investigate the effect of the characteristics of the hydrophilic coating of poly(alkylcyanoacrylate) nanoparticles in their bioadhesiveness using a series of nanoparticles coated with different polysaccharides.

The nanoparticles prepared by two methods of polymerization also differed by the conformation of the polysaccharide chains grafted at the surface of the nanoparticles allowing for the first time to investigate the effect of this parameter on the bioadhesion properties of the nanoparticles ${ }^{17}$.

\section{Materials and methods:}

\subsection{Materials:}

Isobutylcyanoacrylate (IBCA), was used as monomer and was kindly provided as a gift by Loctite (Dublin, Ireland). Dextran 66900 g/mol, were purchased from Sigma (Saint-Quentin Fallavier, France). Dextran $40000 \mathrm{~g} / \mathrm{mol}$ and chitosan medium molecular weight were purchased from Fluka (Saint-Quentin Fallavier, France). 9-anthracenyl methacrylate was purchased from Biovalley (Conches, France). All chemicals were reagent grade and used as purchased. 
Bertholon et al.. J Nanosci Nanotechnol. 2006;6(9-10):3102-9. doi: 10.1166/jnn.2006.418.

\subsection{Preparation of chitosan of various molecular weights:}

2.2.1 Depolymerization of chitosan: Chitosan was selectively depolymerized by reaction with sodium nitrite at various concentrations ${ }^{18}$. Typically, $100 \mathrm{~mL}$ of a solution of chitosan $(2 \%)$ in acetic acid (6\%) was depolymerized during 1 hour with $10 \mathrm{~mL}$ of sodium nitrite, which concentration was either 0.04 or $0.08 \mathrm{~mol} / \mathrm{L}$, at room temperature and under magnetic stirring. Chitosan was precipitated by raising the $\mathrm{pH}$ to 9 with sodium hydroxide. The precipitate was recovered by filtration and washed extensively with acetone before being dried. Chitosan dissolved in acetic acid (0.1 M) was further purified by dialysis against water during 24 hours. The resulted solutions were freeze dried before storage. The degree of deacetylation of the parent chitosan was $79 \pm 2 \%$. According to the literature 19,20 , it is not modified by depolymerization reaction performed with nitrites.

2.2.2 Measurement of molecular weight: The molecular weight of chitosan was determined from capillary viscosity measurements. Briefly, the reduced viscosity of solutions of chitosan of various concentrations $(0.1$ to $2.5 \mathrm{~g} / \mathrm{L})$ in acetic acid $0.1 \mathrm{M}, \mathrm{NaCl} 0.2 \mathrm{M}$ was measured in a Ubbelohde tube (53710/1 Schott Geräte) at $25^{\circ} \mathrm{C}$ (Bath CT1450 Schott Geräte and cooling system CK100 Schott Geräte) using a viscometer AVS400 (Schott Geräte). The intrinsic viscosity $[\eta]$ was then deduced from the reduced viscosity measured for each solution of chitosan by extrapolation at zero concentration. The molecular weight (M) was determined by using the Mark Houwink Sakurada equation: $[\eta]=\mathrm{K}^{*} \mathrm{M}^{\mathrm{a}}$, with $\mathrm{K}=1.81^{*} 10^{-3}$ and $\mathrm{a}=0.93^{21}$.

\subsection{Preparation of nanoparticles:}

2.3.1 Redox radical emulsion polymerization (RREP): The radical polymerization was carried out according to ${ }^{22,23}$. Briefly, dextran or chitosan $(0.1375 \mathrm{~g})$ was dissolved in $4 \mathrm{~mL}$ of nitric acid $(0.2 \mathrm{M})$ at $40^{\circ} \mathrm{C}$ under gentle stirring and argon bubbling. After 10 minutes, $1 \mathrm{~mL}$ of cerium (IV) ammonium nitrate $\left(8^{*} 10^{-2} \mathrm{M}\right.$ in nitric acid $\left.0.2 \mathrm{M}\right)$ and $0.250 \mathrm{~mL}$ of IBCA were successively added. Argon bubbling was maintained for another $10 \mathrm{~min}$ and the reaction was continued for $50 \mathrm{~min}$. Preparations were cooled to room temperature. When preparations were done with dextran, $0.63 \mathrm{~mL}$ of trisodium citrate dihydrate dissolved in water (1.02M) was added to the polymerization medium. The $\mathrm{pH}$ of all suspensions was then adjusted to 7.0 with $\mathrm{NaOH} 1 \mathrm{~N}$. In preparations performed with chitosan, the addition of trisodium citrate induced bulk precipitation of the nanoparticles and the $\mathrm{pH}$ was directly adjusted to 7.0 with $\mathrm{NaOH} 1 \mathrm{~N}$. 
Bertholon et al.. J Nanosci Nanotechnol. 2006;6(9-10):3102-9. doi: 10.1166/jnn.2006.418.

2.3.2 Anionic emulsion polymerization (AEP): The polymerization media was prepared by dissolving at $40^{\circ} \mathrm{C}$ Chitosan $(0.1375 \mathrm{~g})$ in $5 \mathrm{~mL}$ of nitric acid $(0.2 \mathrm{M})$, whose $\mathrm{pH}$ was lower than 1. IBCA $(0.250 \mathrm{~mL})$ was added under strong magnetic stirring and left to polymerize at $40^{\circ} \mathrm{C}$ during $1 \mathrm{~h}$. Chitosane and IBCA concentrations were the same than in RREP but these polymerizations were performed in the absence of ceric ions to avoid the radical polymerization.

2.3.3 Labeling of nanoparticles with Rhodamine : 9-anthracenyl methacrylate (Rhod) was dissolved in acetonitrile at a concentration of $4 \mathrm{mg} / \mathrm{mL}$. Polymerization in presence of Rhodamine labeled monomer was achieved in the dark. In all cases, $1 \mathrm{~mL}$ of Rhod solution was added two minutes after adding IBCA and reaction was let to continue following method without Rhod.

\subsection{Purification of nanoparticles:}

All the polymer suspensions were purified by dialysis (Spectra/Por ${ }^{\circledR}$ membrane 100000 g/mol molecular weight cut off (MWCO), Biovalley, Marne la Vallée, France) two times $1 \mathrm{~h} 30$ and one time overnight against $500 \mathrm{~mL}$ of distilled water. The purified suspensions were stored at $4^{\circ} \mathrm{C}$ until use.

\subsection{Nanoparticles characterization:}

2.5.1 Particle size: The diameter of the nanoparticles was measured at $20^{\circ} \mathrm{C}$ by quasi-elastic light scattering using a Nanosizer N4 PLUS (Beckman-Coulter, Villepinte, France) operating at the angle of $90^{\circ} \mathrm{C}$. The samples were diluted in Milli Q water by $1 / 300(\mathrm{v} / \mathrm{v})$ for nanoparticles made by RREP and by $1 / 150(\mathrm{v} / \mathrm{v})$ for nanoparticles formed by AEP $\mathrm{pH} 1$. The results were expressed as the average of the mean hydrodynamic diameter of the dispersed particles obtained from three determinations. The standard deviation of the size distribution and the polydispersity index were also given. The polydispersity index given by the apparatus is equivalent to the variance of the log-normal distribution. A polydispersity index lower than 0.1 indicates a monodisperse dispersion.

2.5.2 Zeta potential: The electrostatic surface charge of the polymer particles was deduced from the electrophoretic mobility using a Zetasizer nanoseries Nano 2S (Malvern Instruments Ltd., Orsay, France). Dilution of the suspensions (1/200 (v/v)) was performed in $\mathrm{NaCl} 1 \mathrm{mM}$. 
Bertholon et al.. J Nanosci Nanotechnol. 2006;6(9-10):3102-9. doi: 10.1166/jnn.2006.418.

2.5.3 Concentration of nanoparticles in suspension: Concentration of nanoparticles was evaluated by gravimetric measurement after freeze-drying of a known amount of suspension. For freeze-drying, the suspensions were frozen at $-18^{\circ} \mathrm{C}$ and freeze-dried during $48 \mathrm{~h}$ (Christ Alpha 1-4 freeze dryer, bioblock Scientific, Illkrich, France) without using cryo-protecting agent. If the weighted amounts were insufficient, suspensions were concentrated by evaporation of water with a breath of air prior to sampling and lyophilization.

\subsection{Ex-vivo study of bioadhesion:}

Adsorption experiments were performed using a method developed by Durrer et al. ${ }^{15}$. The fresh small intestine of sacrificed male Wistar rats (Charles River, L'Arbresle, France) was excised, rinsed with physiological saline solution ( $\mathrm{NaCL} 0,9 \%$ ), and cut into segments of $5 \mathrm{~cm}$ length. Each segment was opened lengthwise along the mesentery with scissors and spread on an aluminum plate. Another plate of aluminum with a slit (surface $2 \mathrm{~cm}^{2}$ ) in the center was fixed on the mucosa sample.

For bioadhesive experiments, $500 \mu \mathrm{L}$ of each solution of rhodamine labeled nanoparticles was deposited in the split and covered with an aluminum sheet to protect from light. After one hour, the suspension was removed and mucosa was washed three times with $500 \mu \mathrm{L}$ of phosphate buffer ( $\mathrm{pH}$ ) during one minute to remove nonadsorbed nanoparticles. After washing, mucosa was scraped from intestinal wall for dosage. Concentrations of nanoparticles in tested suspensions used were 5,10 and $20 \mathrm{mg} / \mathrm{mL}$ and all experiments were repeated 3 or 4 times

\subsection{Dosage of nanoparticles by fluorimetry:}

Mucosa with nanoparticles were dissolved with $3 \mathrm{~mL}$ of a solution of sodium dodecyl sulfate (SDS) $2 \%$ and $\mathrm{NaOH} 1 \%$ by ultrasound during 2 hours and on planetary agitation overnight. A calibration curve was obtained from sample containing $500 \mu \mathrm{L}$ of nanoparticules suspensions of various concentrations ranging from 0.5 to $20 \mathrm{mg} / \mathrm{ml}, 100 \mathrm{mg}$ of mucus with 3 $\mathrm{mL}$ of a solution of SDS $2 \%$ and $\mathrm{NaOH} 1 \%$. Treatment of calibration samples was the same as for the treatment of experimental samples.

Assays were made with a fluorimeter (spectrometer L550B, Perkin Elmer, Norwalk, USA), the emission wavelength was fixed at $575 \mathrm{~nm}$ and the excitation wavelength at $555 \mathrm{~nm}$ corresponding to the fluorescence characteristics of Rhodamine. Concentration of nanoparticles in mucus was deduced from the calibration curve. 
Bertholon et al.. J Nanosci Nanotechnol. 2006;6(9-10):3102-9. doi: 10.1166/jnn.2006.418.

\subsection{Statistical analysis:}

The results obtained were statistically analyzed by using Mann-Whitney's t-test with a 95\% confidence level $(p<0.05)$ or a $90 \%$ confidence level $(p<0,1)$.

\section{Results and discussion:}

In this study, bioadhesive properties of PIBCA nanoparticles coated with different polysaccharides (dextran, chitosan) were investigated to better understand the influence of the surface properties of nanoparticles on their bioadhesion to intestinal epithelium. In this purpose, ex-vivo bioadhesion experiments were achieved on rat intestinal membrane according to a previously described technique ${ }^{15}$. The core of the nanoparticles has been labeled with rhodamine and the amounts of adhering nanoparticles were determined by fluorimetry.

\subsection{Characteristics of the nanoparticles}

Nanoparticles were obtained by two methods of polymerization (anionic and radical) leading to the formation of amphiphilic copolymers made of hydrophobic PIBCA blocks and hydrophilic polysaccharidic blocks which structure differ depending on the method of polymerization ${ }^{24}$. With the radical redox emulsion polymerization (RREP) nanoparticles coated with either dextran or chitosan where produced whereas only nanoparticles coated with chitosan were prepared by the anionic emulsion polymerization (AEP). The nanoparticles which formed showed a size range between $210 \mathrm{~nm}$ to $575 \mathrm{~nm}$. In agreement with previous results ${ }^{23}$, diameters of nanoparticles mainly depended on molecular weight of the polysaccharide. This may be explained by an increase of the polysaccharide layer thickness at the surface of the nanoparticles as the molecular weight of the polysaccharide increases. Nanoparticles of almost the same size were obtained with and without Rhodamine by the RREP while the diameter slightly increased for the nanoparticles prepared by AEP. For a same molecular weight of the polysaccharide, the size of the nanoparticles depended on the charge of the polysaccharide and on the method of polymerization. The higher diameter was obtained with nanoparticles prepared by RREP with chitosan. The zeta potential of the nanoparticles was clearly affected by the type of the polysaccharide whereas no effect of the method of polymerization could be highlighted in agreement with previous work ${ }^{23}$. The introduction of Rhodamine in the nanoparticles did not affect the zeta potential of the nanoparticles (table 1). 
Bertholon et al.. J Nanosci Nanotechnol. 2006;6(9-10):3102-9. doi: 10.1166/jnn.2006.418.

Table 1: Properties of nanoparticles with and without labeling with rhodamine in pure water for diameter measurements and in $\mathrm{NaCl} 1 \mathrm{mM}$ solution for zeta potential measurements

\begin{tabular}{|l|l|l|l|l|}
\hline & \multicolumn{2}{|l|}{ Without Rhodamine } & \multicolumn{2}{l|}{ With Rhodamine } \\
\hline & $\begin{array}{l}\text { Diameter } \\
(\mathrm{nm})\end{array}$ & $\begin{array}{l}\text { Zeta potential } \\
(\mathrm{mV})\end{array}$ & $\begin{array}{l}\text { Diameter } \\
(\mathrm{nm})\end{array}$ & Zeta potential $(\mathrm{mV})$ \\
\hline Dex 40k - RREP & $246 \pm 25$ & $-9.2 \pm 0.6$ & $225 \pm 22$ & $-8.0 \pm 0.1$ \\
\hline Dex 67k - RREP & $272 \pm 27$ & $-5.0 \pm 0.4$ & $275 \pm 27$ & $-4.6 \pm 0.3$ \\
\hline Chito 30k - RREP & $286 \pm 29$ & ND & $271 \pm 27$ & $+30.3 \pm 0.1$ \\
\hline Chito 70k - RREP & $365 \pm 36$ & $+42.0 \pm 0.7$ & $344 \pm 34$ & $+36.5 \pm 0.2$ \\
\hline Chito 200k - RREP & $575 \pm 57$ & $+43.0 \pm 0.3$ & $550 \pm 55$ & $+48 \pm 1.0$ \\
\hline Chito 30k - AEP & $210 \pm 21$ & $+40.3 \pm 0.5$ & $260 \pm 26$ & $+42.9 \pm 0.3$ \\
\hline Chito 70k - AEP & $251 \pm 25$ & $+42.4 \pm 0.4$ & $340 \pm 34$ & $+44.4 \pm 0.3$ \\
\hline
\end{tabular}

ND : not determined

According to recent studies, Electronic Paramagnetic Resonance (EPR) spectroscopy showed that conformation of polysaccharide chains grafted at nanoparticles surface was different in function of method of polymerization (Figure 1). Dextran at the surface of nanoparticles obtained by RREP can fold at a molecular weight of $18,000 \mathrm{~g} / \mathrm{mol}$ and chitosan at a molecular weight of $100,000 \mathrm{~g} / \mathrm{mol}$ whereas in the case of the nanoparticles prepared by AEP the conformation of the polysaccharide chains at the surface of nanoparticles didn't allow the complete folding of polysaccharide chains whatever the molecular weight of the polysaccharide was ${ }^{17,25}$.

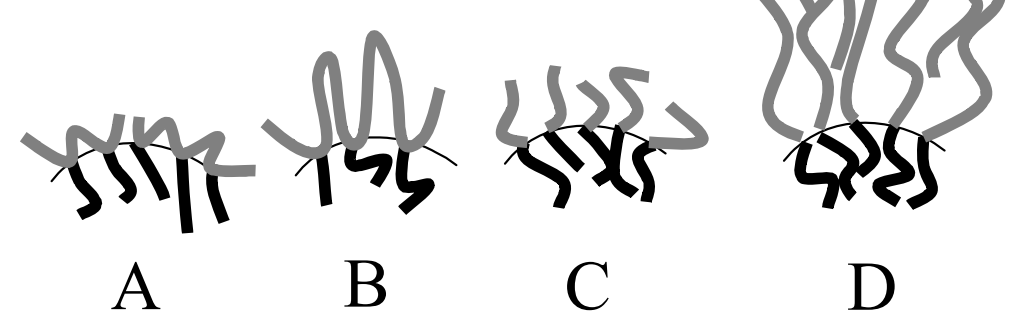

Figure 1: Hypothesized configuration of polysaccharide chains at the surface of nanoparticles prepared either by AEP with polysaccharide of low molecular weight (A) or high molecular weight $(B)$ or RREP with polysaccharide of low molecular weight $(C)$ or high molecular weight (D). Polysaccharide chains: gray line, PIBCA chains: black line. 
Bertholon et al.. J Nanosci Nanotechnol. 2006;6(9-10):3102-9. doi: 10.1166/jnn.2006.418.

\subsection{Effect of the size of the nanoparticles on bioadhesion}

Mucoadhesion of nanoparticles have been described previously ${ }^{13}, 15$. When a particulate suspension is placed in contact with an intestinal mucosa, it has been shown that mucoadhesion was a two steps mechanism, involving primarily the diffusion of the particles from the suspension to the mucosal surface and secondly, the attachment of the particles to the mucosa. As shown previously with model polystyrene particles, the kinetic of adhesion depended mainly on the size of the particles and thus on their diffusion coefficient in the suspending medium. Size decrease resulted in faster adhesion. Whatever the kinetics, adhesion was maximal after 10-20 min for particles in the range of 200-600 nm and did not vary anymore for larger contact times. The second step, corresponding to particles attachment to the mucosa is thought to depend on many parameters and its intensity has been related to various physico-chemical factors. The present study aims to investigate more particularly these adhesion factors explaining why all adhesion experiments were performed at an incubation duration of 1 hour. This incubation time was chosen to avoid any influence of the size of the nanoparticles which is predominant during the step of diffusion of the particles in the suspension medium but before their adhesion on the membrane. Under these conditions, it was assumed that the intensity of adhesion which was measured solely depended on the processes of attachment at the mucosal surface.

During the bioadhesion process and when the particles enter in contact with the mucosal surface, the particles firstly encounter a mucus layer, which can be compared to a thick hydrogel beside the size of the particles. It has been shown previously that the diffusibility of the nanoparticles in this hydrogel depended strongly on the size of the particles ${ }^{6}$. As expected, the attachment of the nanoparticles depended on the diameter of our nanoparticles. The number of particles found in mucus layer was plotted against the diameter of the nanoparticles for all the particles included in this study (Figure 2). Whatever the initial concentration of the suspensions placed in contact with the intestinal mucosa was, the number of particles attached to the mucosa increased considerably when the particle size decreased. However, at low concentration (Figure 2A), dextran-coated nanoparticles exhibited less adhesion than the corresponding chitosan-coated nanoparticles. When the particle concentrations of the suspensions increased (Figure $2 \mathrm{~B}, \mathrm{C}$ ), the number of nanoparticles adsorbed tended to follow the same evolution for all the type of nanoparticles, particularly at $20 \mathrm{mg} / \mathrm{mL}$. This evolution was in agreement with the work of Norris et al., which showed that the permeability of particles through mucus layers followed similar exponential curves ${ }^{6}$. 
Bertholon et al.. J Nanosci Nanotechnol. 2006;6(9-10):3102-9. doi: 10.1166/jnn.2006.418.

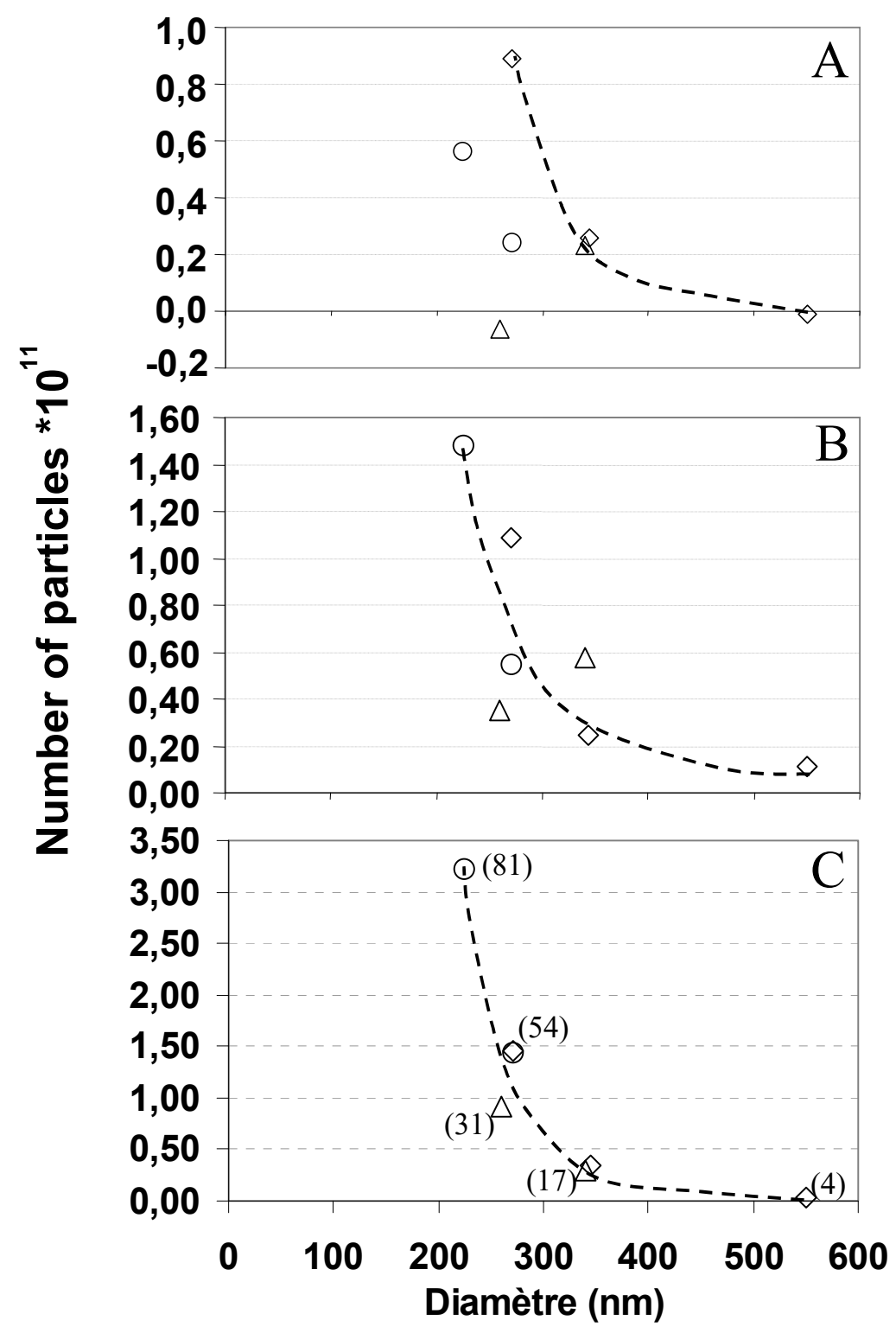

Figure 2: Number of particles fixed on $2 \mathrm{~cm}^{2}$ of mucosa after washing. Nanoparticles made by RREP (circle, diamond) and AEP (triangle) in presence of dextran (circle) and chitosan (diamond, triangle) A: $5 \mathrm{mg} / \mathrm{ml}, B: 10 \mathrm{mg} / \mathrm{ml}, \mathrm{C}: 20 \mathrm{mg} / \mathrm{ml}$, in brackets calculated number of nanoparticles layer at $20 \mathrm{mg} / \mathrm{ml}$. All experiments were repeated 3 or 4 times and for clarity standard deviation were not superimposed.

Furthermore, it was found that the $575 \mathrm{~nm}$ nanoparticles diffused very poorly, in agreement with the calculations of permeability coefficients in the mucus for nanoparticles in this size range ${ }^{16}$. However, these curves reflected not only the diffusion of the particles in the mucus layer, but also their immobilization at different sites of attachment. This was clearly shown by 
Bertholon et al.. J Nanosci Nanotechnol. 2006;6(9-10):3102-9. doi: 10.1166/jnn.2006.418.

the fact that dextran-coated nanoparticles prepared by RREP behaved differently from the other particles (compare figure $2 \mathrm{~A}$ and $2 \mathrm{~B}$ ).

The hypothesis of a diffusion of the particles in the mucus hydrogel was suggested by the following calculations. When considering the number of nanoparticles adherent to the mucus, the surface occupied by the particles (considering a square packing of the particles) has been calculated and further divided by the experimental surface offered by the intestinal fragment. It resulted from this calculation for the experiments made at the highest concentration of nanoparticles $(20 \mathrm{mg} / \mathrm{L})$ that the amount of adhering nanoparticles would require the formation of many layers of particles in the mucus. The calculated number of layers ranged from 4 to 81 (figure 2C), suggested that nanoparticle diffusion inside mucus layer necessarily occurred to represent the experimentally determined amounts of adsorbed nanoparticles. Indeed, if nanoparticles didn't diffuse into the gel, nanoparticles at the surface would have been removed during the washing process following adhesion experiments and multi-layer adsorption could be highlighted. This profile can be linearized (Figure 3) with a logarithm function $\left(\mathrm{R}^{2}=0,9635\right)$, leading to the following equation which represents the adsorption of the nanoparticles as a function of their diameter:

$$
\mathrm{N}=5,9 * 10^{12} * \mathrm{e}^{(-0,0145 \mathrm{D})}
$$

with $\mathrm{N}$ : number of nanoparticles adsorbed in mucus layer and $\mathrm{D}:$ diameter of nanoparticles.

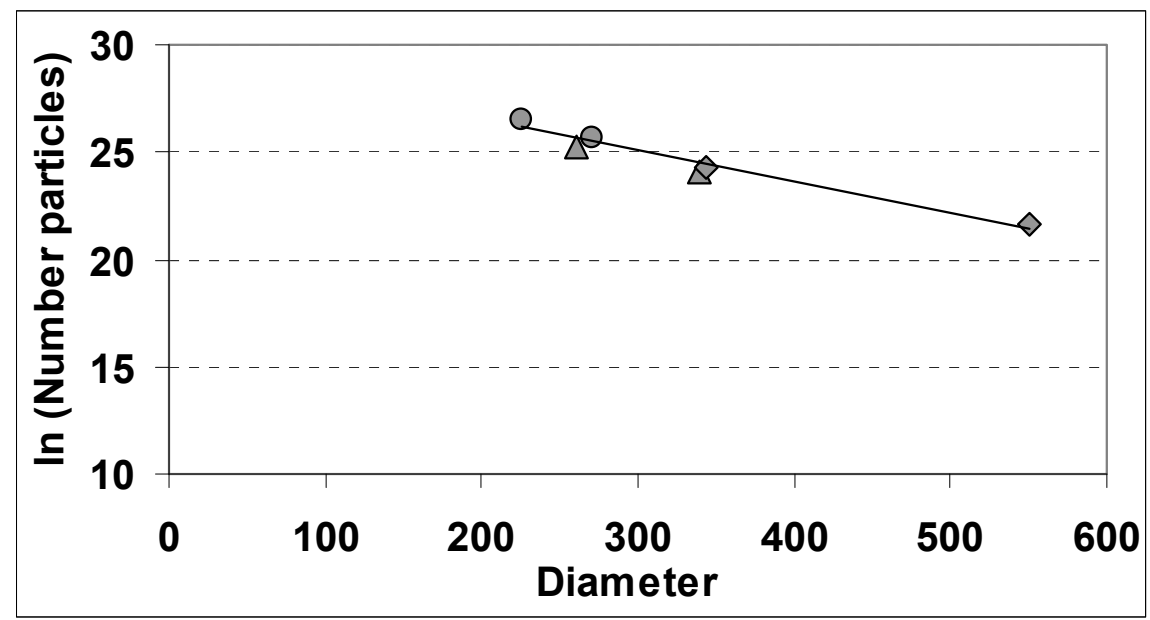

Figure 3: Linearization of evolution of number of nanoparticles absorbed on mucus layer in function of diameter by representation of $\ln$ (number nanoparticles) in function of diameter for nanoparticles obtained by RREP in presence of dextran (circle) or chitosan (diamond) or obtained by AEP in presence of chitosan (triangle), 
Bertholon et al.. J Nanosci Nanotechnol. 2006;6(9-10):3102-9. doi: 10.1166/jnn.2006.418.

Additionally, these results suggested that the possibility for the smallest particles to strongly diffuse in the mucus layer allows the particles to reach much more attachment sites compared to the one available at the mucus layer surface, resulting in very efficient adhesion. However, from a practical point of view, one has to keep in mind that the number of particles adhering to the mucosa is not the most relevant parameter in drug delivery. Indeed for pharmaceutical applications, the most important is the amount of adhering particles expressed in mass which defines the amount of encapsulated and immobilized drug at the nanoparticle surface.

\subsection{Influence of the surface properties on bioadhesion intensity}

Because it was assumed that the duration of the contact between the particle suspensions and the mucosa was high enough (1 hour), it was expected that adhesion had reached equilibrium at the end of adhesion experiments. For further analysis, the amounts of particles which adhere on the mucosa were plotted as isotherms. As can be seen on figures 4, isotherms highlighted differences between nanoparticles depending on their surface characteristics.

Figure 4 A depicts the adhesive behavior of nanoparticles coated with dextran and prepared by RREP. Amounts of adhering particles continued to increase and no plateau could be reached at the highest concentration of nanoparticles in the bulk suspensions $(20 \mathrm{mg} / \mathrm{L})$. At this concentration, adhesion reached about $9 \mathrm{~g} / \mathrm{m}^{2}$, which corresponded to the highest adhesion values obtained in this study. The quasi-linearity of the isotherms suggested that the adhesion sites were still not saturated with these nanoparticles. The effect of the molecular weight of dextran was moderate.

Figure $4 \mathrm{~B}$ shows the adhesion isotherms of chitosan-coated nanoparticles prepared by RREP. Compared to dextran-coated nanoparticles, the adhesion of the chitosan coated nanoparticles increased rapidly at low concentration of the suspensions and a pseudo-plateau was reached for a concentration of $10 \mathrm{mg} / \mathrm{mL}$. This suggested that particles adhesion sites were rapidly saturated. In contrast to dextran-coated nanoparticles, the molecular weight of chitosan influenced the adhesion of the nanoparticles on the mucosa. At the maximal concentration tested $(20 \mathrm{mg} / \mathrm{L})$, the amounts of adherent nanoparticles were about 8 and $4 \mathrm{~g} / \mathrm{m}^{2}$ for chitosan $30000 \mathrm{~g} / \mathrm{mol}$ and $70000 \mathrm{~g} / \mathrm{mol}$, respectively.

Figure $4 \mathrm{C}$ shows the adhesion isotherms of chitosan-coated nanoparticles prepared by anionic polymerization. Although the general form of the isotherms was not very precisely defined, due to the limited number of experimental points, it can be seen that the isotherms were quite similar to those observed for the nanoparticles prepared by radical polymerization. 
Bertholon et al.. J Nanosci Nanotechnol. 2006;6(9-10):3102-9. doi: 10.1166/jnn.2006.418.

Pseudo-plateau could be obtained and the molecular weight of chitosan seemed to modulate the adhesion of the particles to the mucus. The amounts of particles adsorbed were typically lower than $5 \mathrm{~g} / \mathrm{m}^{2}$ whatever the concentrations of the bulk suspensions placed in contact with the intestinal mucosa were. Even, at the concentration of $5 \mathrm{mg} / \mathrm{L}$, the amount of nanoparticles which adsorbed on mucosa was almost nil.

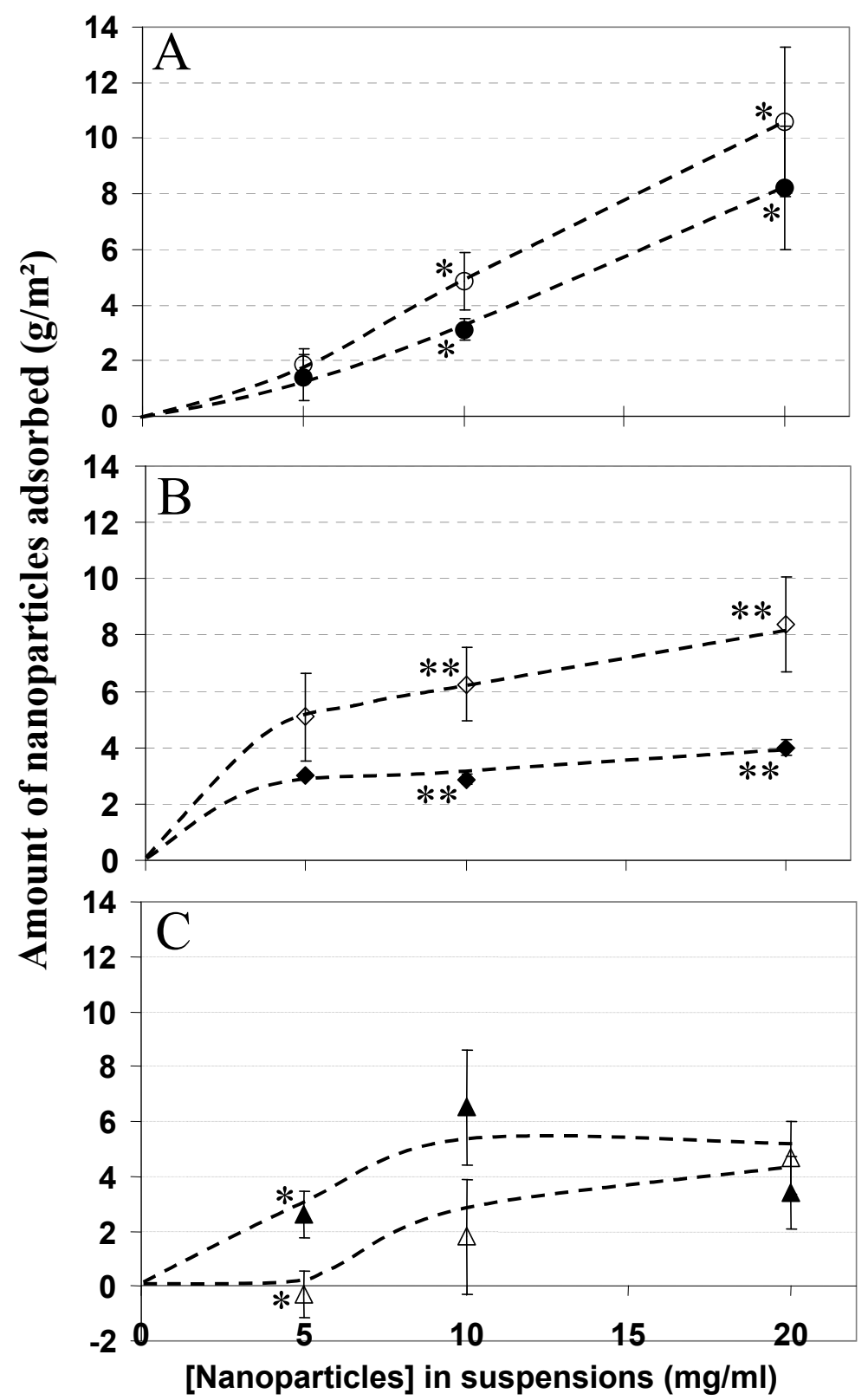

Figure 4: Adsorption isotherms of nanoparticles obtained by RREP: A, B or AEP: $C$, in presence of dextran: A or chitosan: B,C. Open symbol : low molecular weight $(40,000 \mathrm{~g} / \mathrm{mol}$ for dextran, 30,000 $\mathrm{g} / \mathrm{mol}$ for chitosan), black symbol : medium molecular weight (67,000 $\mathrm{g} / \mathrm{mol}$ for dextran, 70,000 $\mathrm{g} / \mathrm{mol}$ for chitosan); Influence of molecular weight statistics * : $p<0,1 ; * *: p<0,05$ 
Bertholon et al.. J Nanosci Nanotechnol. 2006;6(9-10):3102-9. doi: 10.1166/jnn.2006.418.

Differences between these nanoparticles can be attributed to both the nature and the conformation of polysaccharide chains at the surface of nanoparticles. The nature of the adhesive bonds which formed could depend on the type of polymer. Glycoproteins of mucus are negatively charged ${ }^{26}$ and allowed electrostatic interactions with positively charged compounds like chitosan at the surface of the nanoparticles. The number of negative charges in the mucus is finite so does the number of interacting sites with positively charged substances. This can explain the pseudo-plateau shown by the adhesion isotherms observed with the nanoparticles coated with chitosan, which could correspond to a saturation of the negatively charge interacting sites of the mucus. In addition, the intensity of the interaction as evaluated at the plateau level was unexpectedly low in the case of the high molecular weight chitosan compared to the low molecular weight chitosan (Figure 4B). It can be assumed that a higher local viscosity in the high molecular weight chitosan corona can result in a lower accessibility of the interaction sites with the mucus and therefore to a lower adhesion of the nanoparticles. In the case of dextran which is a neutral polysaccharide, no electrostatic interactions can be expected. Although the intimate adhesion mechanism with glycoproteins is unknown, this is in favor to an enhanced diffusion of the dextran-coated nanoparticles in the mucus gel. Indeed, adhesion was in this case concentration dependant (Figure 4A). The marked differences in adhesion profiles for chitosan-coated and dextran-coated nanoparticles clearly supported that adhesion was due to the superimposition of different mechanisms.

The conformation of the chains at the surface of the particles depended on the preparation method. For the nanoparticles prepared by RREP the polysaccharide chains which are grafted at the surface of the nanoparticles in a end-on configuration formed a brush at the surface of the nanoparticles while in the AEP, polysaccharide chains grafted in the side-on configuration formed loops and trains at the nanoparticle surface (figure 1). It is likely that loops of chitosan at the surface of nanoparticles prepared by AEP were quite compact and could restrain interpenetration and interaction of polysaccharide chains with the network formed by the glycoprotein chains of the mucus. This effect could lower the adhesive interactions. Obviously, when the molecular weight of chitosan increased, the length of loops also increased, which could facilitate to some extent the interactions between mucus and nanoparticles prepared by AEP. This can explain that a higher adhesion with the mucosa was observed when chitosan molecular weight increased. For nanoparticles made by RREP, chitosan chains were extended at the surface of nanoparticles and were likely to interact freely 
Bertholon et al.. J Nanosci Nanotechnol. 2006;6(9-10):3102-9. doi: 10.1166/jnn.2006.418.

with the glycoproteins of the mucus layer. It can be suggested that chains interpenetration occurred more easily in this case.

In summary, not only the size, but also the nature and conformation of the polysaccharides at the surface of nanoparticles influenced the adhesion of the nanoparticles on the mucus layer. Adhesion of the nanoparticles to the intestinal mucus can be viewed as a complex process, including the diffusion of the particles in the hydrogel, depending on their size, followed by their interactions with the mucus glycoproteins. It is likely that the molecular interactions played a key role in this latter process. For getting a better understanding of these effects, differences in polymer chain flexibility and local modification of viscoelasticity should probably be considered. Indeed, as shown previously at a macroscopic scale, these polymers can interact with mucus. For example, the viscoelasticity of mucus can be decreased in presence of dextran as shown elsewhere using the airway mucus ${ }^{27}$. Dextran was also found to diffuse through mucus layer in rat distal colon mucus ${ }^{28}$. However, a more detailed investigation of the dynamic of polymer chains at the nanoparticle surface would be required for being able to fully describe these effects. Finally, from a practical point of view, it is interesting to point out that the bioadhesive properties of the nanoparticles could be adjusted to some extent by varying the nature of the coating polymer and probably its conformation.

\section{Conclusion:}

Influence of the coating of nanoparticles by polysaccharides with varying molecular weight and molecular conformation on their bioadhesive properties has been investigated. In agreement with the literature, accumulation of the smallest nanoparticles in the mucus layer could be attributed to a diffusion mechanism. Further, interaction of nanoparticles with mucus was promoted by electrostatic interactions in the case of chitosan coated nanoparticles and non electrostatic interactions in the case of dextran coated nanoparticles. Interestingly, differences in bioadhesion were observed, which depended on the nature of the polysaccharide and its conformation at the surface of the nanoparticles, suggesting that bioadhesive properties of nanoparticulate systems can be modulated by a fine tuning of the surface properties of the particles.

\section{Aknowledgements}

Authors wish to thank K. Broadley from Loctite (Dublin, Ireland) for providing the isobutylcyanoacrylate used in the preparation of the nanoparticles and Irene Bravo-Usuna for 
Bertholon et al.. J Nanosci Nanotechnol. 2006;6(9-10):3102-9. doi: 10.1166/jnn.2006.418.

helpful discussion. I. Bertholon-Rajot was a fellow from the French Ministery of Research. This work was part of the Integrated Action Program (Amadeus Program 06645SH).

\section{References}

1. M. Thanou, J. C. Verhoef and H. E. Junginger, Adv Drug Deliv Rev 52, 117 (2001)

2. A. Vila, A. Sanchez, M. Tobio, P. Calvo and M. J. Alonso, J Control Release 78, 15 (2002)

3. C.-M. Lehr, J. A. Bouwstra, E. H. Schacht and H. E. Junginger, Int J Pharm 78, 43 (1992)

4. S. Barbault-Foucher, R. Gref, P. Russo, J. Guechot and A. Bochot, J Control Release 83, 365 (2002)

5. G. Ponchel and J. Irache, Adv Drug Deliv Rev 34, 191 (1998)

6. D. A. Norris and P. J. Sinko, J Appl Polym Sci 63, 1481 (1997)

7. Y. Huang, W. Leobandung, A. Foss and N. A. Peppas, J Control Release 65, 63 (2000)

8. N. G. M. Schipper, K. M. Varum, P. Stenberg, G. Ocklind, H. Lennernas and P. Artursson, Eur J Pharm Sci 8, 335 (1999)

9. S. A. Agnihotri, N. N. Mallikarjuna and T. M. Aminabhavi, J Control Release 100, 5 (2004)

10. M. Garcia-Fuentes, C. Prego, D. Torres and M. J. Alonso, Eur J Pharm Sci 25, 133 (2005) 11. N. G. M. Schipper, S. Olsson, J. A. Hoogstraate, A. G. deBoer, K. M. Varum and P. Artursson, Pharm Res 14, 923 (1997)

12. G. Ponchel, M. Montisci, A. Dembri, C. Durrer and D. Duchene, Eur. J. Pharm. Biopharm. 44, 25 (1997)

13. C. Durrer, J. M. Irache, F. Puisieux, D. Duchene and G. Ponchel, Pharm Res 11, 674 (1994)

14. C. Durrer, J. Irache, D. Duchene and G. Ponchel, J. Colloid Interface Sci. 170, 555 (1995)

15. C. Durrer, J. M. Irache, F. Puisieux, D. Duchene and G. Ponchel, Pharm Res 11, 680 (1994)

16. D. A. Norris, N. Puri and P. J. Sinko, Adv Drug Deliv Rev 34, 135 (1998)

17. C. Chauvierre, C. Vauthier, D. Labarre and H. Hommel, Colloid Polym Sci 282, 1016 (2004)

18. M. Huang, E. Khor and L. Y. Lim, Pharm Res 21, 344 (2004)

19. G. G. Allan and M. Peyron, Carbohydr Res 277, 257 (1995)

20. G. G. Allan and M. Peyron, Carbohydr Res 277, 273 (1995)

21. T. Khan, K. Peh and H. Ch'ng, J Pharm Pharm Sci 3, 303 (2000)

22. C. Chauvierre, D. Labarre, P. Couvreur and C. Vauthier, Macromol. 36, 6018 (2003)

23. I. Bertholon-Rajot, D. Labarre and C. Vauthier, Polymer 46, 1407 (2005)

24. I. Bertholon, S. Lesieur, D. Labarre, M. Besnard and C. Vauthier, Submitted

25. I. Bertholon, D. Labarre, H. Hommel and C. Vauthier, Submitted

26. N. A. Peppas and Y. Huang, Adv Drug Deliv Rev 56, 1675 (2004)

27. W. Feng, S. Nakamura, E. Sudo, M. M. Lee, A. Shao and M. King, Pulm Pharmacol Ther 12, 35 (1999)

28. L. Szentkuti, J Control Release 46, 233 (1997) 